\title{
Long term results after surgical basal cell carcinoma excision in the eyelid region
}

Stefan Pieh, Andreas Kuchar, Philipp Novak, Rainer Kunstfeld, Gunda Nagel, F J Steinkogler

\begin{abstract}
Aims-To evaluate the data for patients with basal cell carcinoma (BCC) in the eyelid region, to demonstrate histologically controlled tumour excision, and to prove the efficacy of the treatment on the basis of long term observations.

Methods-Retrospective analysis of 382 microscopically controlled BCC excisions in the eyelid apparatus (350 patients) in a follow up study over 5.7 (SD 1.1) years. Tumour location, tumour size, and histological results were recorded. The same procedure was followed for recurrences. Follow up examinations were carried out $1,3,6$, and 12 months after the operation, and then annually for a further 4 years or longer.

Results-A recurrence rate of $5.36 \%$ was observed after the primary operation. $60.3 \%$ of first recurrences occurred in the medial canthus, $41.2 \%$ showed in depth extension, and sclerosing types were overly represented at $35.3 \%$. After the second operation the recurrence rate increased to $14.7 \%$ and reached $50 \%$ after a third and fourth operation.

Conclusions-The greatest risk of recurrence exists for BCCs of the medial canthus with in depth extension, and for sclerosing types. The recurrence rate increases after every operation. For high risk cases, consideration should be given to adjuvant treatment such as radiotherapy.

(Br f Ophthalmol 1999;83:85-88)
\end{abstract}

Department of

Ophthalmology,

University of Vienna,

Medical School,

Vienna, Austria

S Pieh

A Kuchar

P Novak

G Nagel

F J Steinkogler

Department of

Dermatology,

University of Vienna,

Medical School,

Vienna, Austria

R Kunstfeld

Correspondence to: Stefan Pieh, MD, University

Eye Clinic Vienna,

Währinger Gürtel 18-20,

A-1090 Wien, Austria.

Accepted for publication 31 July 1998 using zinc chloride paste was too destructive for periocular tissues ${ }^{11}$ and was therefore replaced by cryosurgery. Free autologous skin transplants are necessary for covering the wounds resulting from removal of extended tumours. The literature describes several methods for ensuring primary healing of the transplant. The technique using pressure bandages was introduced in 1966 by Mustarde, ${ }^{12}$ and in 1979 Metha $^{13}$ developed a suture technique for free transplants that secures the contact with the wound bed. The introduction in the early 1980s of fibrin sealing for skin transplants ${ }^{14}{ }^{15}$ was a fundamental innovation which has since become the standard technique for wound covering of free autologous skin transplants.

\section{Subjects and methods}

PATIENTS AND TUMOUR CLASSIFICATION

This study involved the evaluation of 382 radical BCC excisions in the eyelid region (350 patients) carried out between 1980 and 1993. Fifty two of these patients underwent primary surgical tumour excision at other hospitals and were then assigned to our hospital for treatment of the recurrences. Clinical and macroscopic diagnosis was carried out. In cases of widespread and infiltrating tumours the extension was verified by echography, computed tomography, and magnetic resonance imaging for purposes of planning the operation.

Tumour localisation was classified as upper or lower lid and medial or lateral canthus. The BCCs were divided according to macroscopically or echographically measured tumour size into the groups 1-4 mm, 5-9 $\mathrm{mm}, 10-14 \mathrm{~mm}$, $15-19 \mathrm{~mm}$, and $20 \mathrm{~mm}$ or larger. Tumours growing deeply into the orbicularis oculi muscle are strictly classified as BCCs with an in depth extension. Histological results were divided into three groups corresponding to the literature. ${ }^{16}{ }^{17}$ The histological patterns of BCC are determined by the relative amounts of cellular and stromal elements and by the extent of cellular differentiation.

\section{First group}

Nodular BCC, clinically the most common type of BCC, is characterised by relatively undifferentiated cells and tumour masses containing a small proportion of connective tissue. Nodular BCC appears histologically in a solid, keratotic or adenoidal form. In solid types the peripheral cell layer of the tumour mass shows a characteristic palisade arrangement. Keratotic types are characterised by parakeratotic cells and horn cysts in addition to undifferentiated cells. The adenoid BCC resembles glandlike structures and the tumour has a mucoid appearance. 
Second group

Sclerosing (morphoea-like) BCC-The histological pattern is determined by a high proportion of fibrous stroma. Tumour cells form strands which often penetrate deeply into the dermis. Furthermore, sclerosing BCC is characterised by the loss of palisade formation, especially in the deeper lying parts of the tumour.

\section{Third group}

Superficial multicentric BCC shows irregular proliferation of tumour tissue attached to the underside of the epidermis. In most cases there is little penetration into the dermis. The peripheral cell layer of the tumour formation shows palisading.

\section{SURGICAL TECHNIQUE}

The operation was performed under local anaesthesia in 375 cases; general anaesthesia was necessary in six cases because of the extent of the tumour. Our technique corresponds to the principles of Mohs's micrographic surgery. ${ }^{1018} 19$ The first step is resection of the macroscopically visible tumour. The next critical step is the excision of thin layers of tissue circumferential to the debulking defect. All specimens must be marked with sutures in order to indicate their in situ position. The specimens are taken to the histopathological laboratory where frozen sections tangential to the surgical margin are prepared using a microtome and then stained. The results of microscopic examination are reported to the operating theatre as quickly as possible and used for deciding whether additional tissue resections are necessary. Wound closure should not be performed until total removal of the tumour has been verified histologically. The desired primary skin closure after tumour excision may be achieved by undermining the wound margins if the suture tension does not restrict the eyelid function. If the tumour was extensive, primary skin closure is often impossible. In such cases we used skin transplants from the upper lid or the postauricular region.

\section{POSTOPERATIVE EXAMINATION}

Routinely, macroscopic clinical examinations were carried out $1,3,6$, and 12 months after the operation. Further examinations took place annually for a further 4 years or longer. The mean follow up period for the patients was 5.7 (SD 1.1) years.

\section{Results}

The ratio of female to male patients was $1.4: 1$ (204 and 146). The mean age of the female patients was 69.0 years; that of the male patients was 67.1 years.

The primary tumour locations were distributed as follows: 196 cases $(56.0 \%)$ on the lower lid, 95 cases $(27.1 \%)$ on the medial canthus, 29 cases $(8.3 \%)$ on the upper lid, 30 cases $(8.6 \%)$ on the lateral canthus (Fig 1A).

The size of the BCCs at diagnosis were distributed as follows: $1-4 \mathrm{~mm}$ in $19.1 \%$ (57 patients), 5-9 $\mathrm{mm}$ in $40.9 \%$ (122 patients), $10-14 \mathrm{~mm}$ in $20.5 \%$ (61 patients), $15-19 \mathrm{~mm}$

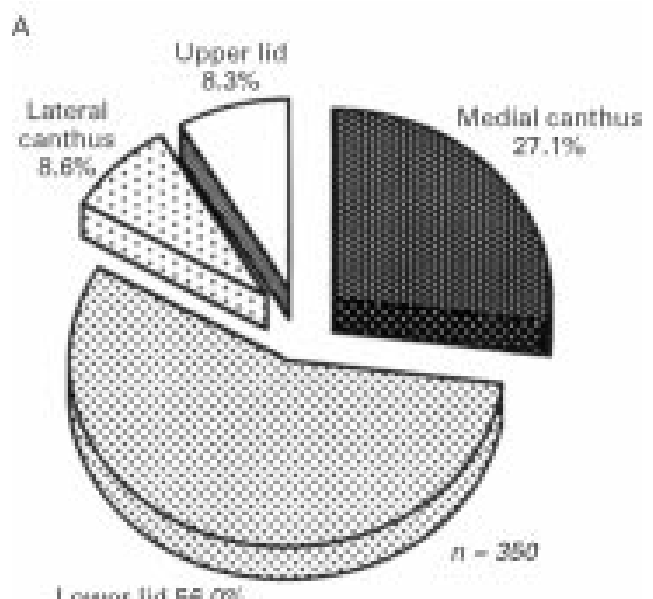

Lowar lid 56.09 .
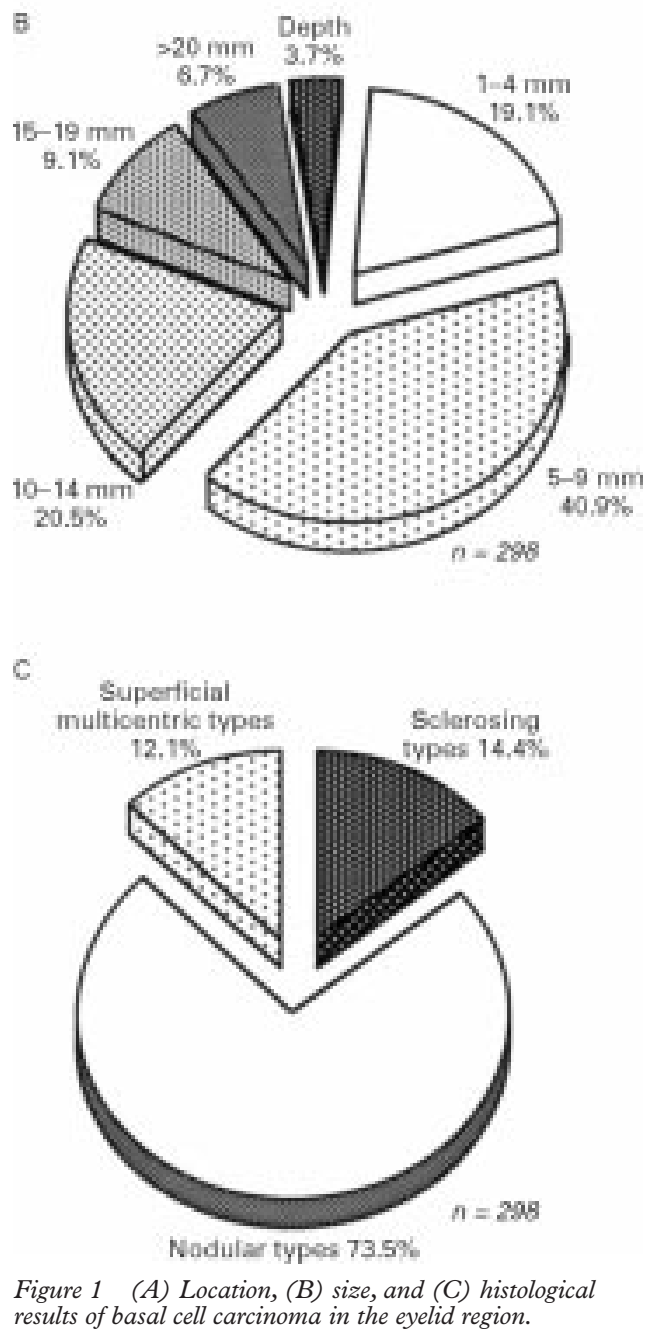

results of basal cell carcinoma in the eyelid region.

in $9.1 \%$ (27 patients), $20 \mathrm{~mm}$ or more in $6.7 \%$ (20 patients), BCC with in depth extension in $3.7 \%$ (11 patients) (Fig 1B).

The histological types of the primary tumour were distributed as follows: sclerosing in $14.4 \%$ (43 patients), nodular types in $73.5 \%$ (219 patients), and superficial multicentric types in $12.1 \%$ (36 patients) (Fig 1C).

Primary wound closure was possible in 333 cases. In this group neither wound infections nor other complications of wound healing were 
observed. In the remaining 49 cases the wound covering was performed by free autologous transplant using the fibrin sealing technique. This method was simple to use. Complications such as infection were not observed.

For the group of 298 cases whose primary operation was performed at our hospital, the recurrence rate was $5.36 \%$ (16 patients).

The locations of the 68 first recurrences (16 patients first operated at our hospital and 52 patients referred to our hospital for first recurrent surgery) were distributed as follows: $60.3 \%$ (41 patients) on the medial canthus, $22.0 \%$ (15 patients) on the lower lid, $11.8 \%$ (eight patients) on the upper lid, 5.9\% (four patients) on the lateral canthus (Fig 2A).

Of these first recurrences, $41.2 \%$ (28 patients) showed an in depth extension, $4.4 \%$ (three patients) had a tumour size of $1-4 \mathrm{~mm}$, $8.8 \%$ (six patients) of $5-9 \mathrm{~mm}, 14.7 \%$ (10 patients) of $10-14 \mathrm{~mm}, 14.7 \%$ (10 patients) of $15-19 \mathrm{~mm}$ and $16.2 \%$ (11 patients) of $20 \mathrm{~mm}$ or greater (Fig 2B).

Histological analysis of the 68 first recurrences revealed the sclerosing type of BCC in $35.3 \%$ (24 patients), nodular types in $55.9 \%$ (38 patients), and superficial multicentric types in $8.8 \%$ (six patients) (Fig 2C).

In the group of 68 secondary operations the recurrence rate was $14.7 \%$ (10 patients). In seven of these 10 patients the recurrent tumour was located on the medial canthus, in five cases the tumour possessed an in depth extension and seven of these 10 recurrences were classified as sclerosing.

In the 10 patients requiring a third operation, a recurrence was diagnosed in four cases. Three of these four recurrences were in the medial canthus, two showed an in depth extension, and all were of the sclerosing type.

In the final group of four patients requiring a fourth operation, two recurrences occurred during the follow up period, were located on the medial canthus, showed an in depth extension, and were classified as sclerosing.

\section{Discussion}

The introduction of microscopically controlled tumour excision by Mohs in 1941 and the further development of the cryogenic technique have led to considerable improvements in surgical tumour excision. According to previously published reports, residual tumours remain in the margins of resection after up to $50 \%$ of surgical BCC excisions performed without histological control. ${ }^{2021}$

The great majority of patients with a BCC in the lid region are over 60 years old, the most common location being the lower lid. In most of the cases the tumour size at diagnosis was flat and between 5 and $9 \mathrm{~mm}: 73 \%$ of the BCCs are histologically classified as nodular types.

Our follow up period involving examinations 1, 3, 6, and 12 months after the operation and then annually for a further 4 years, is in accordance with the literature recommendation. ${ }^{22}$

A recurrence rate of $5.36 \%$ was observed for the 298 patients undergoing histologically con-
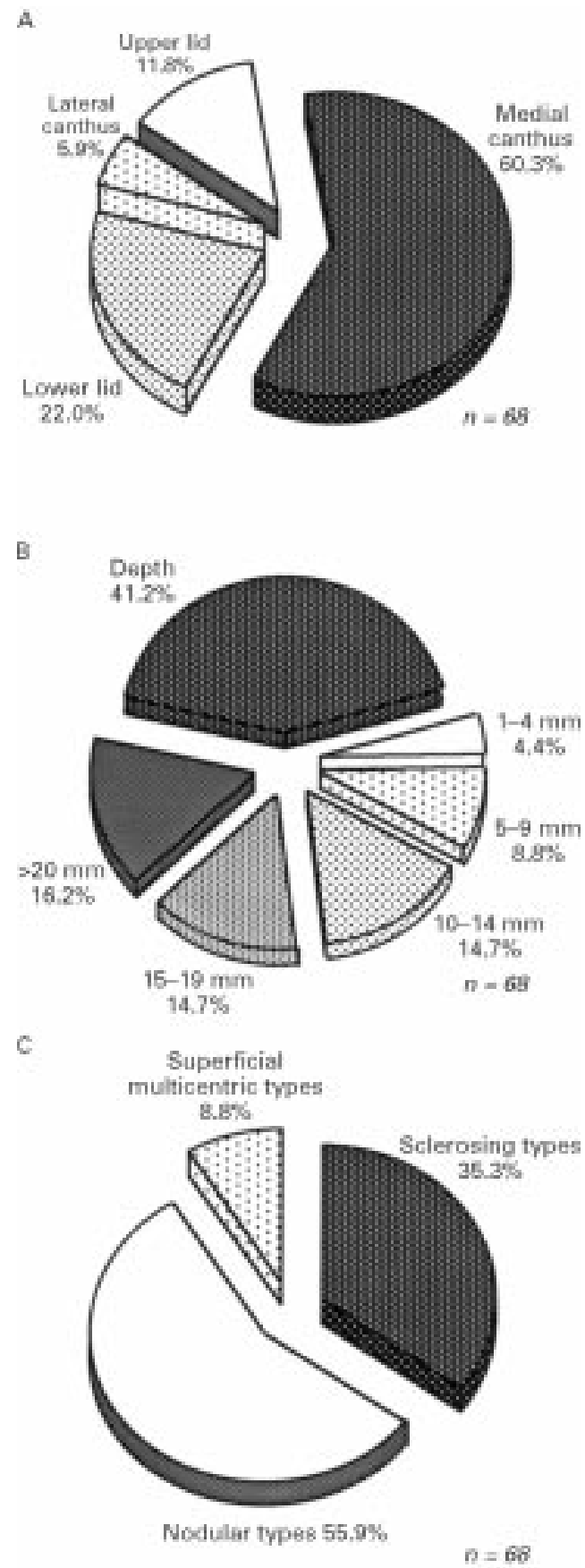

Figure 2 (A) Location, (B) size, and (C) histological results of basal cell carcinoma recurrences in the eyelid region.

trolled tumour resection in our hospital. Close cooperation with the pathologist and uninterrupted preparation of the layered sections are important factors in ensuring that the histological results can be relied on. Surgical instruments which have previously been used for tumour debulking must not be reused. Excision of the layered segments must be carried out using a separate set of instruments.

It is evident from the group of 68 first recurrences that a BCC on the medial canthus is more likely to recur than one located anywhere else in the lid region (Fig 2A). This may be due to the complex anatomy of the medial canthal 
tendon, of the canalicular system, and of the orbital septal attachments. Not only are these predisposed to early in depth extension of the tumour, but the lacrimal drainage system induces the surgeon to be more cautious during tumour excision than is the case at other locations in the lid region. Analysis of the tumour extension also reveals that most recurrences are associated with in depth extension (Fig 2B). A possible explanation would be that precise excision of a thin layer of tissue for microscopic control is easier at the periphery than underneath the debulking defect resulting from primary tumour excision.

A total of $14.4 \%$ of the group of histologically evaluated primary tumours were sclerosing BCCs compared with $35.3 \%$ of the group of 68 primary recurrences. This indicates that the sclerosing type has a higher potential for recurrence compared with other types.

After the first reoperation, the recurrence rate increases from $5.36 \%$ to $14.7 \%$. This may indicate that the recurrent tumours consist of cells with a greater invasive potential. This "surgical selection" of aggressive tumour cell types would also explain the increase in the recurrence rate after the second and third reoperation. The evaluation of recurrences after the first reoperation confirms that the greatest risk of recurrence exists for BCCs in the medial canthus, for those with an in depth extension, and for the sclerosing type. The recurrence rate increases to $50 \%$ for patients who have undergone a second or third operation. Analysis of these recurrences confirms yet again the critical factors of medial canthus, in depth extension, and sclerosing type.

The fibrin sealing technique proved to be an easy method for fixing autologous transplants and since its introduction has replaced other techniques such as pressure bandages ${ }^{12}$ and sutures. ${ }^{13}$ In addition to the practical advantages of the fibrin sealing technique, good cosmetic results have also been achieved.

In our opinion microscopic control of the wound margins ought to become standard practice in the surgical treatment of BCC. Correct diagnosis at an early stage permits tumour eradication in accordance with Mohs, preserving functionally important tissues, and allowing primary wound closure with resultant simplification of the surgical procedure. Routine ophthalmological examinations should therefore be used as an opportunity to detect lesions in the eyelid region, especially with patients over 60 . It should be borne in mind that surgical excision of a BCC in the medial canthus or with in depth extension is a sophisticated procedure. This applies particularly to recurrence excisions. In high risk cases-for example, a recurrent operation in the medial canthus with a histologically evaluated sclerodermiform type confirmed by the primary excision-the application of adjuvant radiotherapy should be considered.

1 Aurora AL, Blodi FC. Lesions of the eyelid. A clinicopathological study. Surv Ophthalmol 1970;15:94-104.

2 Vitaliano PP, Urbach F. The relative importance of risk factors in nonmelanoma carcinoma. Arch Dermatol 1980;116: $454-6$.

3 Lober CW, Fenske NA. Basal cell, squamous cell and sebaceous gland carcinomas of the periorbital region. $f \mathrm{Am}$ .

4 Margo CE, Waltz K. Basal cell carcinoma of the eyelid and periocular skin. Surv Ophthalmol 1993;38:169-92.

5 Doxanas MT, Green WR. Factors in the successful surgical management of basal cell carcinoma of the eyelid. Am $\mathfrak{f}$ management of basal cell carci

6 Torre D. Cryosurgery of eylid tumors. In: Jakobiec FA, ed. Ocular and adnexal tumors. Birmingham, AL: Aesculapius, 1978:517-24.

7 Fitzpatrick PJ, Jamieson DM, Thompson GA, et al. Tumors of the eyelids and their treatment by radiotherapy. Radiology 1972;104:661-5.

8 Saccini V, Lovo GF, Arioli N. Carbon dioxide laser in scalp tumor surgery . Laser Surg Med 1987;7:6-11.

9 Wilson BD, Mang TS, Stoll H. Photodynamic therapy for treatment of basal cell carcinoma. Arch Dermatol 1992;128 1597-601.

10 Mohs F. Chemosurgery: microscopically controlled method of cancer excision. Arch Surg 1941;42:279-95.

11 Mohs F. The chemosurgical method for the microscopically controlled excision of external cancer with reference to the eyelid. Trans Am Acad Ophthalmol Otorayngol 1958;62:335.

12 Mustarde JC. Repair and reconstruction in the orbital region. Edinburgh: Livingstone, 1966:39-43.

13 Metha H. Surgical management of carcinoma of eyelids and periobital skin. Br f Ophthalmol 1979;63:578-85

4 Steinkogler FJ. Fibrin tissue adhesive for the repair of lacerated canaliculi lacrimales. In: Schlag G, Redl H, eds. Fibrin sealant in ophthalmology and neurosurgery. Vol 2. Berlin: Springer, 1986:92-7.

15 Steinkogler FJ. Lid split surgery and fibrin sealing of free skin transplants. Ophthalmic Plast Reconstr Surg 1986;2: $183-7$.

16 Fitzpatrick TB, Johnson RA, Wolf K, et al. Dermatology in general medicine. 4th ed. pp 840-7.

17 Walter F. Lever histopathology of the skin. 7th ed. pp 622-34. 8 Roenigk RK. Mohs' micrographic surgery. Mayo Clin Proc 1987;63:175-83.

9 Swanson NA. Mohs surgery: technique, indications, applications and the future. Arch Dermatol 1983;119:761-73.

20 Einaugler RB, Henkind P. Basal cell epithelioma of the eyelid: apperant incomplete removal. Am $\mathcal{F}$ Ophthalmol 1969;67:413-17.

21 Rakofsky SI. The adequacy of surgical excision of basal cell carcinoma. Ann Ophthalmol 1973;5:596-600.

22 Tijl JW, Koorneff L. The optimal follow-up time for basal cell carcinoma of the eyelid. Doc Ophthalmol 1990;75:275-9. 